\title{
KOVALEVSKA VS. KOVACIC-TWO DIFFERENT NOTIONS OF INTEGRABILITY AND THEIR CONNECTIONS
}

\author{
PAWEE GOLDSTEIN \\ Department of Mathematics, Informatics and Mechanics, University of Warsaw \\ Banacha 2, 02-097 Warszawa, Poland \\ E-mail: goldie@mimuw.edu.pl
}

Ordinary differential equations all share the same common root-real physical problems. But, although the physical motivation remains the most important one, the way the subject develops does depend highly on the methods available. In the exposition I would like to show some connections between two methods of checking the ODE for integrability (whatever it should mean), with distant motivations and techniques. These are the so-called Painlevé tests and the methods originating in Ziglin's theory and developed widely by Morales and Ramis.

1. Integrability of an ordinary differential equation. When should we consider an ODE integrable? And what does the term: 'to integrate' mean here? The answer to this question depends deeply on the mathematical background of the person answering (and, of course, on the equation itself).

First, we should restrict ourselves to some 'good' class of ODE.

If we are to use any algebraic methods, the demand to have the ODE:

$$
E\left(u^{(n)}, u^{(n-1)}, \ldots, u, x\right)=0
$$

algebraic in $u$ and its derivatives and analytic in $x$ seems natural.

The most basic information on the solution we can obtain from Cauchy theorem: the solution does exist locally (except at some singular points). Of course, to call an ODE integrable, we would require much more: we need some global information on the general solution.

Here the theories part. Suppose the equation does allow a Hamiltonian formulation. From the Hamiltonian point of view we would state the equation integrable if a sufficient

2000 Mathematics Subject Classification: Primary 34M55; Secondary 34M35.

The paper is in final form and no version of it will be published elsewhere. 
number of first integrals in involution did exist (Liouville integrability) ${ }^{1}$. If the equation happens to be linear, we do have the differential Galois approach: it is integrable, if it can be integrated in (generalized) quadratures.

Possibly the most general and geometric point of view originates in the work of Paul Painlevé: an ODE is integrable, if its general solution is a function (i.e. is a single-valued map). As this is a very demanding condition (even linear equations may fail to fulfill it: e.g. the equation $2 x u^{\prime}+u=0$ has as the general solution $\left.u(x)=c x^{1 / 2}\right)$, we should correct it as follows:

An ODE is considered integrable if its general solution is uniformizable (can be made single-valued by either extending its domain to a suitable Riemann surface, or, equivalently, restricting it to the complex plane with some Fuchsian cuts). The uniformizability of the general solution of an ODE will be called from now on the Painlevé property and abbreviated (PP).

2. The Painlevé property. Some important remarks should be made here about the definition of the PP. First thing that the reader should be aware of is that a nonlinear ODE, if it cannot be transformed to the form $u^{(n)}(x)=F\left(u^{(n-1)}(x), \ldots, u(x), x\right)$, may have, apart from the general solution, some (lower dimensional) families of the so-called singular solutions, that are not reductions of the general solution. A classical example is the Clairaut equation (here in its simplest form):

$$
\frac{1}{2}\left(u^{\prime}\right)^{2}+x u^{\prime}-u=0
$$

with $u(x)=A x+\frac{1}{2} A^{2}$ as a general solution and $u(x)=-\frac{1}{2} x^{2}$ as the singular one.

The Painlevé methods do not consider at all the singular solutions; the reason for this will be explained in a while.

Another thing is to realize what type of obstacles to the PP we are likely to meet in the world of 'good' ODE. To answer this question, we must recall that a singular point of a solution may be critical (causing multivaluedness) or noncritical. As we saw above, even solutions of linear ODE may have critical singular points. Happily, in the linear ODE case, we may a priori, without solving the equation, state where to look for these singularities (and cut them out from our $\mathbb{C}$ plane together with some line segments) - they may occur only at the singular points of the coefficients of the equation.

It is not so with nonlinear ODE. A very simple example: $u^{\prime} u=1$ does have a general solution $u(x)=\sqrt{2\left(x-x_{0}\right)}$ - thus with a branching point at arbitrary point $x_{0}$, depending on the initial values. This type of singularity, the location of which is impossible to determine without imposing some initial constraints, is called a movable singularity. If an equation does have movable, critical singular points, it surely cannot have the PP. It is not hard to notice that it is the only obstruction; this leads to another definition of the PP:

An ODE has the PP if it does not have movable critical singularities.

${ }^{1}$ The problem is, of course, not that simple even in this case. The questions are: 1 . How regular would we require these first integrals to be? 2. What domain should we take for our equation (as the regularity of the first integrals may depend on it)? For a deeper discussion see [19]. 
3. The programme of $\mathbf{P}$. Painlevé. The first motivation to study equations having PP came with a deep result of Painlevé, Poincaré and Fuchs ([28], [24]) stating that among the solutions of all first order ODEs $E\left(u^{\prime}, u, x\right)=0$ with $E$ polynomial in $u^{\prime}$ and $u$ and analytic in $x$ there is only one new transcendental function (i.e. a single-valued mapping) defined: the Weierstrass elliptic $\wp$ function.

The next question given by Painlevé ([25], [26]) was: What if we take the ODE of degree two, then three, etc.? Are there new functions defined? Is it possible to classify $\mathrm{all}^{2}$ the first, second, third order ODE with a single-valued general solution?

This is the famous Painlevé programme. It has been completed in order 2 by Painlevé ([25], [26], [27]) and his student Gambier ([9]). They obtained 53 equation types, of which 47 were integrable in terms of known (including elliptic) functions, while 6 defined new functions, known as the Painlevé transcendents:

$$
\begin{aligned}
(P 1) \quad u^{\prime \prime}= & 6 u^{2}+x \\
(P 2) \quad u^{\prime \prime}= & 2 u^{3}+x u+\alpha \\
(P 3) \quad u^{\prime \prime}= & \frac{\left(u^{\prime}\right)^{2}}{u}-\frac{u^{\prime}}{x}+\frac{\alpha u^{2}+\beta}{x}+\gamma u^{3}+\frac{\delta}{u}, \\
(P 4) \quad u^{\prime \prime}= & \frac{\left(u^{\prime}\right)^{2}}{2 u}+\frac{3}{2} u^{3}+4 x u^{2}+2\left(x^{2}-\alpha\right) u+\frac{\beta}{u}, \\
(P 5) \quad u^{\prime \prime}= & {\left[\frac{1}{2 u}+\frac{1}{u-1}\right]\left(u^{\prime}\right)^{2}-\frac{u^{\prime}}{x}+\frac{(u-1)^{2}}{x^{2}}\left[\alpha u+\frac{\beta}{u}\right]+\gamma \frac{u}{x}+} \\
& +\delta \frac{u(u+1)}{u-1}, \\
(P 6) \quad u^{\prime \prime}= & \frac{1}{2}\left[\frac{1}{u}+\frac{1}{u-1}+\frac{1}{u-x}\right]\left(u^{\prime}\right)^{2}-\left[\frac{1}{x}+\frac{1}{x-1}+\frac{1}{u-x}\right] u^{\prime}+ \\
& +\frac{u(u-1)(u-x)}{x^{2}(x-1)^{2}}\left[\alpha+\beta \frac{x}{u^{2}}+\gamma \frac{x-1}{(u-1)^{2}}+\delta \frac{x(x-1)}{(u-x)^{2}}\right] .
\end{aligned}
$$

These new functions, often encountered in contemporary physics, are extensively studied nowadays $([17],[7])$.

With the formulation of Painlevé's programme we can understand why this approach does not bother with singular solutions: they are always solutions of a lower order ODE (see [3], par. 5.1), so they have been considered at an earlier stage of classification.

How did Painlevé obtain his classification? His so-called double méthode (double method) consisted of two parts:

- local study of the equation, in order to determine the necessary conditions for the PP (and, if our equation depends on a number of arbitrary parameters, to sieve out the values of these parameters that satisfy the conditions),

- in cases when the necessary conditions are satisfied - construction and/or study of the general solution in order to prove their sufficiency.

The first part of this method used the following perturbation idea:

${ }^{2}$ With respect to a transformation group consisting of holomorphic changes of $x$ and homographic transformations of $u$, to which the PP is invariant (see [3]). 
Consider an ODE of degree $N$, in Poincaré canonical form:

$$
u^{\prime}=E(u, x, \varepsilon), \quad x \in \mathbb{C}, \quad u \in \mathbb{C}^{n}, \quad \varepsilon \in \mathbb{C}
$$

where $\varepsilon$ is a small parameter, $E$ is holomorphic in some domain $D \ni\left(u_{0}, x_{0}, 0\right)$. We may then formally expand both sides of (1) in the perturbation parameter $\varepsilon$ :

$$
\sum_{n=0}^{\infty} \varepsilon^{n} \frac{\mathrm{d}}{\mathrm{d} x} u^{(n)}=\sum_{n=0}^{\infty} \varepsilon^{n} E^{(n)}\left(u^{(n)}, u^{(n-1)}, \ldots, x\right)
$$

We obtain in this way an infinite sequence of equations, of which only the first one is nonlinear. The whole sequence is equivalent to equation (1).

TheOrem 1 (Painlevé, Bureau). With the above assumptions, if the general solution of (1) is single-valued in $D$ except possibly at $\varepsilon=0$, then it is single-valued also at $\varepsilon=0$; moreover the $u^{(n)}$ are single-valued for all $n$.

The version above is due to Painlevé, the result of Bureau, slightly stronger, says that the theorem is valid with without movable critical points in place of single-valued.

How can this serve to classify unperturbed equations? Painlevé, having a $2^{\text {nd }}$ order equation written as a system of two $1^{\text {st }}$ order ones (in canonical form) introduced the perturbation parameter $\varepsilon$ in the following way:

$$
\begin{aligned}
x & =z_{0}+\varepsilon \zeta \\
u & =\sum_{n=0}^{\infty} \varepsilon^{n+p} u^{(n)} .
\end{aligned}
$$

Substituting it into the equation $E(u, x)=0$ we obtain, after some term rearrangement,

$$
\sum_{n=0}^{\infty} E_{n} \varepsilon^{n+q}=0
$$

and, due to arbitrariness of $\varepsilon, \forall_{n} E_{n}=0$ - a series of equations, often referred to as variational equations.

For $n=0$ the original, unperturbed equation is reproduced. The equation $E_{1}=0$ the first variational equation (linearization of the original equation, équation auxiliaire of Darboux) - is usually much simpler than the original one - and thus easier to solve (or, at least, to check for the PP)-whenever it fails to possess the PP, so does the original one.

4. Meromorphy test of Kovalevska. At the same time as the Painlevé method was developed, Sophie Kovalevska and Paul Hoyer investigated the singularity structure of the general solution by another means. Their method, based on the assumption that the general solution is meromorphic and can be expanded in a Laurent series with a finite singular part, lead Kovalevska to a new solution of the spinning top system. Their method was, of course, less general than the Painlevé approach - it did not take into account the possible existence of movable noncritical essential singularities (which are not an obstruction for the PP); it also could not detect a holomorphic general solution. The method, developed further by Appelrot and Gambier, was made an algorithm in 1980 by Ablovitz, Ramani and Segur [1]. 


\section{The ARS algorithm (a very crude sketch with an example)}

Let us consider $\quad u^{\prime \prime}-2 u^{3}-x u=0, \quad(\mathrm{P} 2)$ equation with $\alpha=0$.

0 . Expand $u$ and the coefficients of the equation around an arbitrary pole $x_{0}$ :

$$
\begin{aligned}
\tau & =x-x_{0} \\
x & =\tau+x_{0} \\
u & =\sum_{n=0}^{\infty} u_{n} \tau^{p+n}
\end{aligned}
$$

1. Determine the leading order exponent $p$-insert the leading terms of the expansions into the equation:

$$
(p-1) p u_{0} \tau^{p-2}+O(p-1)-2 u_{0}^{3} \tau^{3 p}+O(3 p+1)=0
$$

and thus $p=-1$, as the leading order terms must cancel.

2. Determine the leading order coefficient - substitute the value(s) of $p$ into (3) and repeat the cancellation argument to obtain $u_{0}$.

$$
2 u_{0} \tau^{-3}-2 u_{0}^{3} \tau^{-3}+O(-2)=0
$$

thus (as $\left.u_{0} \neq 0\right) u_{0}= \pm 1$.

The next steps should be applied to all the pairs $\left(p, u_{0}\right)$ obtained above. The calculations will follow for $\left(p, u_{0}\right)=(-1,1)$.

3. Calculate the "resonances" - by substituting all the expansions and rearranging the terms we obtain recurrence relations between $u_{n}$ :

$$
(n+1)(n-4) u_{n}=2 \sum_{n_{1}=1}^{n-1} \sum_{n_{2}=0}^{n-n_{1}} u_{n_{1}} u_{n_{2}} u_{n-n_{1}-n_{2}}+2 \sum_{k=1}^{n-1} u_{0} u_{k} u_{n-k}+u_{n-3}+u_{n-2} .
$$

The recurrence formula is well defined except for $n=-1$ and $n=4$ - these values of $n$ are the resonances. The resonance at $n=-1$ is a somewhat mysterious rule ${ }^{3}$. At the other resonance(s) we obtain

4. Compatibility condition(s) - as the lhs of (4) for these values of $n$ is 0 , so should be the rhs.

Step by step, from (4), we obtain $u_{1}=0, u_{2}=-\frac{1}{6}, u_{3}=-\frac{1}{4}$ and we check that the compatibility condition is indeed satisfied. A similar calculation gives for the other branch $\left(u_{0}=-1\right) u_{1}=u_{2}=0, u_{3}=\frac{1}{4}$ and a compatibility condition for $n=4$, also satisfied. For both branches we get thus two arbitrary constants in the expansion $\left(x_{0}\right.$ and $\left.u_{4}\right)$-the number required to construct the general solution.

This algorithm is widely known under the name of Painlevé test ${ }^{4}$ - regardless to deep resentment that Painlevé felt towards this method, considering it too incomplete and

\footnotetext{
${ }^{3}$ The resonances correspond to arbitrary constants, so the resonance at -1 , although there is no compatibility condition there, may be attributed to the arbitrariness of $x_{0}$.

${ }^{4}$ It seems to be a rule with S. Kovalevska results that they are attributed to someone else, cf. Cauchy-Kovalevska theorem.
} 
restrictive. In the last 20 years a great number of improvements and generalizations to the test have been added - the reader should consult a fantastic exposition by R. Conte ([3]).

5. Branching point of ideas. As I mentioned before, the very first application of the meromorphy test gave a new solution of a spinning top motion equations - to add to previously known ones: the isotropic, Euler and Lagrange solutions ([13], see also [3], par. 2.5). Unfortunately, due to the weakness of her method, Kovalevska could not claim that there are no other uniform solutions. There was a possibility of overlooking a solution with a noncritical essential singularity, or even a holomorphic solution - as the meromorphy test was not able to detect it. Five years later Liapunov and Nekrasov [23] proved that indeed no other solutions are uniform.

The approach of Liapunov was particularly ingenious. He perturbed the Hamiltonian equation, like in the method of Painlevé, but instead of considering a general, unknown solution $u$, he took a particular one. Next he analyzed the variational equation (VE) obtained by the perturbation; by analysis of the leading order term he proved that the only cases at which there are no branching points in its solution are the four already known. From the modern point of view his method was nothing but analysis of the monodromy group of the VE. This is justified by the following theorem:

Theorem 2 ([19], Proposition 1). Consider an (autonomous) equation $u^{\prime}=E(u)$ defined on a complex analytic manifold $M$. Let $u(x)$ be its particular, single-valued solution. If the general solution of this equation is single-valued over $\mathbb{C}$ then the monodromy groups of all the variational equations (obtained by perturbation of $u(x)$ ) must be trivial.

This approach was a starting point for S. L. Ziglin, who, in 1982, proved an important theorem on the restrictions imposed on the variational equation of a Hamiltonian system by the existence of first integrals.

He considered a complex Hamiltonian system

$$
u^{\prime}=X_{H}(u),
$$

by perturbing the equation along a particular solution representing a Riemann surface $\Gamma$ he obtained the variational equation (VE)

$$
\dot{\eta}=X_{H}^{\prime}(u(x)) \eta
$$

$\left(\dot{\eta}=\frac{\mathrm{d}}{\mathrm{d} x} \eta\right)$. As the original system had a first integral - the Hamiltonian itself - we can reduce the VE using $\mathrm{d} H(u(x))$; we obtain the so-called normal variational equation (NVE), which is a linear Hamiltonian system. Also any other first integrals that are in involution (with each other and the Hamiltonian) allow us to reduce the VE. He obtained ([31]):

TheOrem 3 (Ziglin). Suppose the $n$-degrees of freedom Hamiltonian system has $k$ first integrals in involution (including the Hamiltonian) and $n-k$ additional meromorphic first integrals, independent of the previous ones on a neighbourhood of $\Gamma$, but not necessarily on $\Gamma$. Let us assume that the monodromy group of the NVE (VE reduced by the $k$ first integrals in involution) contains a non-resonant transformation $g$. Then all the other elements of the monodromy group transform eigendirections of $g$ into eigendirections of $g$. 
The reader should notice that, although the system has $n$ first integrals, only $k$ of them must be in involution - therefore the system does not have to be completely integrable.

A well known fact that the monodromy group is contained in the differential Galois group of a linear ODE, and, if the ODE's singular points are regular-Zariski dense in it, led J. J. Morales-Ruiz to an idea of applying the differential Galois theory into Ziglin's method. His main observation was that Ziglin's conditions on the monodromy group must be connected with the Picard-Vessiot integrability of the VE. His work with J. P. Ramis led to several theorems, of which the basic version is known as the Morales-Ramis theorem ([19],[21],[22]):

Theorem 4 (Morales, Ramis). - Assume that the system (5) has $k$ first integrals in involution and independent in a neighbourhood as an analytic integral curve $C$ (not necessarily on $C$ itself) and that the points at infinity are regular singularities of the VE. Then the identity components of the Galois group of the VE and of the NVE are abelian symplectic groups.

- Ziglin's theorem is valid (mutatis mutandis) with monodromy group replaced by Galois group.

The Morales-Ramis theorem has been successfully applied to a long list of problems, several of them are presented in the book [20] by Morales-Ruiz; still new, important results are obtained, see for example [30]. One of the advantages of this approach is that for the $2^{\text {nd }}$ order ODE there exists an efficient, algorithmic method of computing the Picard-Vessiot extension, provided the equation is integrable; a failure in the algorithm proves the nonintegrability of the ODE.

The method, known as Kovacic's algorithm ([12]), is based on the following observations:

1. A $2^{\text {nd }}$ order ODE can always be transformed to the so-called reduced invariant form:

$$
\xi^{\prime \prime}-g \xi=0
$$

2. The Galois group of the equation $(6)$ is contained in $S L(2, \mathbb{C})$.

3. All the subgroups of $S L(2, \mathbb{C})$ are well known and classified (see, for example, [20]), the only one among them with the identity component not solvable is $S L(2, \mathbb{C})$ itself.

4. The equation (6) might be transformed to a Riccati equation:

$$
v^{\prime}=g+v^{2}
$$

by substitution $v=-(\log \xi)^{\prime}$; the original equation (6) is integrable if and only if the Riccati equation has an algebraic solution.

5. The degree of the minimal polynomial $Q(v)$ is bounded and in the set $1,2,4,6,12$. The algorithm leads to computation of $Q(v)$, the details can be found in [20], [8].

6. An example-Bianchi IX cosmological model. At the end of the presentation I would like to show an interesting example, in which both methods of integrability testing interact in an interesting manner. 
The Bianchi IX cosmological model (mixmaster model) is a homogeneous Einstein manifold. The name (and number) comes from Luigi Bianchi's classification of such homogeneous spaces - there are IX types of them, classified by the Lie algebras of their structural group (see [15]). For this particular one, the Lie algebra is defined by the following commutation rules:

$$
\begin{aligned}
& {\left[X_{1}, X_{2}\right]=X_{3}} \\
& {\left[X_{2}, X_{3}\right]=X_{1}} \\
& {\left[X_{3}, X_{1}\right]=X_{2} .}
\end{aligned}
$$

It was proposed as a model of the early universe by Belinski, Khalatnikov ([2]) and Misner ([18]), who believed that its behaviour is chaotic. Also the first computation of the maximal Liapunov characteristic number (MLCN) - a standard tool to detect chaosgave a positive (affirmative for chaos) result. Unfortunately, more detailed calculations ([11]) gave the MLCN equal to 0, indicating that the system may not be chaotic at all.

The equations of motion in the mixmaster universe (i.e. the geodesic equation) can be written in logarithmic time $t$ :

$$
\begin{aligned}
& \left(\log x_{1}\right)^{\prime \prime}=\left(x_{2}-x_{3}\right)^{2}-x_{1}^{2} \\
& \left(\log x_{2}\right)^{\prime \prime}=\left(x_{3}-x_{1}\right)^{2}-x_{2}^{2} \\
& \left(\log x_{3}\right)^{\prime \prime}=\left(x_{1}-x_{2}\right)^{2}-x_{3}^{2}
\end{aligned}
$$

with a first (energy) integral:

$$
\begin{aligned}
E=-\left(\left(\log x_{1}\right)^{\prime}\left(\log x_{2}\right)^{\prime}\right. & \left.+\left(\log x_{2}\right)^{\prime}\left(\log x_{3}\right)^{\prime}+\left(\log x_{1}\right)^{\prime}\left(\log x_{3}\right)^{\prime}\right)+ \\
& +x_{1}^{2}+x_{2}^{2}+x_{3}^{2}-2\left(x_{1} x_{2}+x_{2} x_{3}+x_{1} x_{3}\right)=0
\end{aligned}
$$

in the absence of matter.

There exist single-valued, closed form solutions:

- a 4-dimensional family of Taub:

$$
x_{1}=\frac{k_{1}}{\sinh \left(k_{1}\left(t-t_{1}\right)\right)}, \quad x_{2}=x_{3}=\frac{k_{2}^{2} \sinh \left(k_{1}\left(t-t_{1}\right)\right)}{k_{1} \sinh ^{2}\left(k_{2}\left(t-t_{2}\right)\right)}
$$

(or cyclically);

- two 3-dimensional families, which are solutions of first order reductions of the system (7): the Darboux-Halphen system ([10], integrable in Hermite modular elliptic functions) and the Euler system (integrable in Jacobi elliptic functions), representing the motion of a rigid body around its centre of mass. I do not write the equations for these two, as I will be interested mostly in the family of Taub.

The first assaults at this system with the tools of Kovalevska analysis proved unsuccessful ([5]) - the authors obtained a triple resonance at $n=-1$ and another triple one at $n=2$, so the ARS algorithm was unable to provide sufficient number of arbitrary constants. No proof of the lack of Painlevé property could be obtained this way either.

A more refined version of the Kovalevska test (perturbative Painlevé approach), originating from Kruskal ([14]) and made an algorithm by Conte, Fordy and Pickering ([4]) 
was used next year by the same authors $([6])$, with much better results - a failure to fulfill the compatibility condition in the general case was noticed.

This fact was not properly understood until A. Latifi, M. Musette and R. Conte published a deeper analysis ([16]), based on the so-called Fuchsian perturbative method (a rigorous version of perturbative approach mentioned before).

This method follows at the beginning the idea of Painlevé explained in section 3 , but with a different way of introducing the parameter $\varepsilon$ than shown in (2):

$$
u=\sum_{n=0}^{\infty} \varepsilon^{n} u^{(n)}, \quad E=\sum_{n=0}^{\infty} \varepsilon^{n} E^{(n)}=0
$$

with $x$ unchanged; moreover, we perturb a known particular solution instead of the general one. Latifi et al. perturbed the Taub solution, obtaining (with $k_{1}=k_{2}=0$ ) the first variational equation:

$$
\frac{\mathrm{d}^{2}}{\mathrm{~d} \tau^{2}} \eta+\left(\frac{2}{\tau^{2}}-\frac{4(\tau-1)^{2}}{\tau^{4}}\right) \eta=0
$$

with $\tau=\frac{t-t_{2}}{t_{1}-t_{2}}, \eta=x_{2}^{(1)}-x_{3}^{(1)}$.

Then a standard Kovalevska (ARS) test is applied. In such a manner we obtain conditions (no-log conditions) assuring no logarithmic behaviour in the singular point if a multiple negative resonance occurred in Kovalevska approach.

These no-log conditions were satisfied by the known Taub and Darboux-Halphen families of solutions, and an unknown yet 4-dimensional family with the solutions of the Euler system as a subfamily.

Local singularity analysis displayed a movable transcendental essential singularitythus proving that the equation did not have PP.

What is special about the above equation is that it is exactly the NVE of the mixmaster system, when the Taub solution is perturbed (in [20] a slightly different equation is obtained, due to a different choice of the particular solution from the Taub family). The Kovacic algorithm fails, which gives us that the Galois group of the NVE is $S L(2, \mathbb{C})$ and the mixmaster system is not completely integrable with rational first integrals as a Hamiltonian system.

We can imagine that the obstruction in both cases was the same: the movable transcendental singularity, which cannot be "tamed" by rational first integrals.

The connections between the perturbative Painlevé methods and the Morales-Ramis approach still need a lot of clarification. The Painlevé methods are widely used by physicists and they evolve quickly, as to suit particular needs. Some of these ideas (weak Painlevé property ([29]), analysis of higher order VE in the perturbative approach) seem closely related to more global analysis given by differential Galois theory. The very interesting paper by J. J. Morales-Ruiz ([19]) gives more problems than answers in this point.

Acknowledgements. The author would like to thank the organizers of the Differential Galois Theory Workshop for their generous invitation and for turning my attention to the topic; Prof. J. J. Morales-Ruiz for interesting discussions and a copy of his results ([19]), and, finally, to Dr Piotr Goldstein, my father and my first teacher of the Painlevé analysis. 


\section{References}

[1] M. J. Ablovitz, A. Ramani and H. Segur, A connection between nonlinear evolution equations and ordinary differential equations of P-type, J. Math. Phys. 21 (1980), 715-721.

[2] V. A. Belinski, I. M. Khalatnikov and E. M. Lifshitz, Oscillatory approach to a singular point in the relative cosmology, Adv. Phys. 19 (1970), 524-574.

[3] R. Conte, The Painlevé approach to nonlinear ordinary differential equations, in: The Painlevé Property: One Century Later (ed. R. Conte), CMR Series in Mathematical Physics, Springer-Verlag, 1999, 77-180.

[4] R. Conte, A. P. Fordy and A. Pickering, A perturbative Painlevé approach to nonlinear differential equations, Physica D 69 (1993), 33-58.

[5] G. Contopoulos, B. Grammaticos and A. Ramani, Painlevé analysis for the mixmaster universe model, J. Phys. A: Math. Gen. 26 (1993), 5795-5800.

[6] G. Contopoulos, B. Grammaticos and A. Ramani, The mixmaster universe model, revisited, J. Phys. A: Math. Gen. 27 (1994), 5357-5361.

[7] B. Dubrovin, Painlevé transcendents in two-dimensional topological field theory, in: The Painlevé Property: One Century Later (ed. R. Conte), CMR Series in Mathematical Physics, Springer-Verlag, 1999, 287-412.

[8] A. Duval, The Kovacic Algorithm with applications to special functions, in: M. Singer (ed.), Differential Equations and Computer Algebra, Academic Press, London, 1991, 113-130.

[9] B. Gambier, Sur les équations différentielles du second ordre et du premier degré dont l'intégrale générale est à points critiques fixes, thesis, Paris, 1909; Acta Math. 33 (1910), $1-55$.

[10] G.-H. Halphen, Sur un système d'équations différentielles, C. R. Acad. Sci. Paris 92 (1881), 1101-1103.

[11] D. Hobill, D. Bernstein, D. Simpkins and M. Welge, in: Proc. 12th Int. Conf. on General Relativity and Gravitation (Boulder, Univ. of Colorado) 1989; Class. Quantum Grav. 8 (1992), 1155-1172.

[12] J. J. Kovacic, An algorithm for solving second order linear differential equations, J. Symb. Comput. 2 (1986) 3-43.

[13] S. Kowalevski, Sur le problème de la rotation d'un corps solide autour d'un point fixe, Acta Math. 12 (1889), 177-232.

[14] M. D. Kruskal, Flexibility in applying the Painlevé test, in: D. Levi, P. Winternitz (ed.), Painlevé Transcendents. Their Asymptotics and Physical Applications, NATO ASI Series, Plenum Press, 1992, 187-196.

[15] L. D. Landau and E.M. Lifshitz, Théorie des champs, Mir, Moscou, 1970.

[16] A. Latifi, M. Musette and R. Conte, The Bianchi IX (mixmaster) cosmological model is not integrable, Phys. Lett. A 194 (1994), 83-92.

[17] D. Levi and P. Winternitz (ed.), Painlevé Transcendents. Their Asymptotics and Physical Applications, NATO ASI Series, Plenum Press, 1992.

[18] C. M. Misner, Mixmaster universe, Phys. Rev. Lett. 22 (1969), 1071-1074.

[19] J. J. Morales-Ruiz, Kovalevskaya, Liapunov, Painlevé, Ziglin and the differential Galois theory, Regul. Chaotic Dyn. 5 (2000), 251-272.

[20] J. J. Morales-Ruiz, Differential Galois Theory and Non-Integrability of Hamiltonian Systems, Birkhäuser, Basel 1999.

[21] J. J. Morales-Ruiz and J. P. Ramis, Galoisian obstructions to integrability of Hamiltonian systems, Methods Appl. Anal. 8 (2001), 33-95. 
[22] J. J. Morales-Ruiz and J. P. Ramis, Galoisian obstructions to integrability of Hamiltonian systems II, Methods Appl. Anal. 8 (2001), 97-111.

[23] P. K. Nekrasov, The problem of motion of a rigid body about a fixed point, Matem. Sb. 16 (1892), 508-517.

[24] P. Painlevé, Leçons sur la théorie analytique des équations différentielles (Leçons de Stockholm), Hermann, Paris, 1897; repr. in: CEuvres de Paul Painlevé, vol. I, Éditions du CNRS, Paris, 1973.

[25] P. Painlevé, Mémoire sur les équations différentielles dont l'intégrale générale est uniforme, Bull. Soc. Math. France 28 (1900), 201.

[26] P. Painlevé, Sur les équations différentielles du second ordre et d'ordre supérieur dont l'intégrale générale est uniforme, Acta Math. 25 (1902), 1-86.

[27] P. Painlevé, Sur les équations différentielles du second ordre à points critiques fixes, C. R. Acad. Sc. Paris 143 (1906).

[28] H. Poincaré, Sur un théorème de M. Fuchs, Acta Math. 7 (1885), 1-32.

[29] A. Ramani, B. Grammaticos and T. Bountis, The Painlevé property and singularity analysis of integrable and non-integrable systems, Phys. Rep. 180 (1989), 159-245.

[30] H. Yoshida, A new necessary condition for the integrability of Hamiltonian systems with a two-dimensional homogeneous potential, Physica D 128 (1999), 53-69.

[31] S. L. Ziglin, Branching of solutions and non-existence of first integrals in Hamiltonian mechanics I, Func. Anal. Appl. 16 (1982), no. 1, 8-23, and II, Func. Anal. Appl. 17 (1983), no. 3, 30-41. 